\title{
A rapid flow cytometric technique for the detection of platelet-monocyte complexes, activated platelets and platelet-derived microparticles
}

\author{
LAURA PEARSON ${ }^{*}{ }^{\dagger}$, JIM THOM ${ }^{*}$, MURRAY ADAMS ${ }^{\dagger}$, ROBERT OOSTRYCK $^{\dagger}$, ROM KRUEGER $^{*}$, \\ GERALD YONG ${ }^{\ddagger}$, ROSS BAKER ${ }^{*}, \S$
}

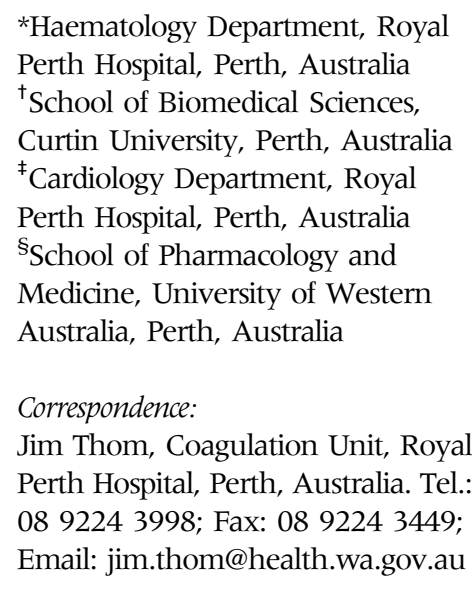

doi:10.1111/j.1751-553X.2008.01059.X

Received 22 August 2007; accepted for publication 11 February 2008

\section{Keywords}

Activated platelets, flow cytometry, percutaneous coronary interven-

tion, platelet-monocyte complexes

\section{SUMMARY}

Platelet activation occurs in a variety of clinical situations in which it directly contributes to the pathology. This study reports a simple flow cytometric assay for platelet activation which measures platelet-derived microparticles, activated platelets and platelet-monocyte complexes. Pre- and post analytical conditions were investigated and optimized and a normal range established on 20 healthy controls. Twenty patients pre- and post percutaneous coronary intervention (PCI) were tested with the technique. Soluble activation markers sCD40 ligand and sPselectin and plasma phospholipid levels were measured in both groups. There was a significant increase in activated platelets and plateletmonocyte complexes between normal and pre-PCI $(P=0.005$ and 0.0275, respectively) suggesting an activated state. There was a significant fall in activated platelets post-PCI $(P=0.0027)$ which was mirrored by a fall in soluble CD40 ligand, soluble P-selectin and plasma phospholipid levels $(P=0.0066,<0.0001$ and 0.0032 , respectively) consistent with antiplatelet therapy administered during the process. This is a reliable and rapid method for the assessment of ex vivo platelet activation which may be an aid in diagnosis and help guide therapy for patients with thrombotic disease.

\section{INTRODUCTION}

Normal platelet function is essential for preventing excessive blood loss and the formation of intravascular thrombi. Historically, platelet function testing has been mainly directed towards detecting hyporeactivity in the investigation of bleeding disorders. However, morbidity and mortality caused by thrombosis far exceeds that as a result of bleeding diatheses.
Platelets are major contributors to the thrombotic process in many common disorders such as acute myocardial infarction (AMI) (Furman et al., 2001a), stable and unstable angina (Vidal et al., 2001), peripheral arterial disease (Cassar et al., 2003) and acute cerebral ischaemia (Zeller, Tschoepe \& Kessler, 1999). A blood test that can reflect in vivo platelet activation is potentially a powerful tool in the clinical laboratory. 
Activation of platelets causes a series of morphological and functional changes including a conformational change in glycoprotein (GP) IIb-IIIa, membrane rearrangement with microparticle formation, expression of neoantigens such as P-selectin and the rapid formation of platelet-leucocyte aggregates (for review see Hickerson and Bode, 2002). These changes can be detected by flow cytometry using a wide range of monoclonal antibodies available as markers of platelets and platelet activation.

Several modifications of flow cytometry for platelet analysis have been reported, including the use of either washed platelets (Wehmeier et al., 1991), platelet rich plasma (Ejim et al., 1990) or whole blood (Shattil, Cunningham \& Hoxie, 1987). As the physiological function of platelets is to respond rapidly to procoagulant stimuli, they are susceptible to artefactual activation following venesection. Whole blood techniques minimize activating platelets by avoiding centrifugation. Some investigators have used red cell lysis to provide clearer delineation of the platelet and white cell populations (Furman et al., 1998; Barnard et al., 2003; Ray et al., 2005). However, it has been reported that lysis may also lead to artefactual activation (Li, Goodall \& Hjemdahl, 1997).

Until recently, surface P-selectin expression (CD62) has been considered as the gold standard marker of platelet activation; however, degranulated platelets lose their surface P-selectin (Michelson et al., 2001). Platelets expressing P-selectin also rapidly adhere to white blood cells via P-selectin glycoprotein ligand-1 (Andre, 2004). The formation of platelet-leucocyte aggregates, particularly platelet-monocyte complexes, has been reported to be a reliable marker of activation (Michelson et al., 2001).

There are two reported approaches for detection of platelet-monocyte complexes. Barnard et al. (2003) lysed whole blood to remove red blood cells, whereas Hagberg and Lyberg (2000) reported a technique with no lysis, centrifugation or washing of samples with reduced potential for inducing ex vivo platelet activation.

In this study, a flow cytometric technique for the evaluation of platelet activation in whole blood samples has been optimized. Artefactual platelet activation has been minimized by avoiding centrifugation, washing, vortexing and red blood cell lysis. Samples have been processed rapidly and fixed with paraformaldehyde after incubation with antibodies. This technique has been used to measure platelet-derived microparticles (PDM), activated platelets expressing CD62 and platelet-monocyte complexes in normal, as well as clinical samples from patients with acute coronary syndrome who underwent early PCI.

These cellular markers have been compared with plasma markers of platelet activation, soluble CD40 ligand and soluble P-selectin. We have also reported a new method, factor X-activated clotting (XACT). This technique is sensitive to procoagulant phospholipid present in patient plasma and is based on the capacity of a sample to shorten the clotting time of phospholipid depleted plasma (Exner et al., 2003).

\section{MATERIALS AND METHODS}

\section{Ethics}

Use of clinical samples for this study were approved by the ethics committee of Royal Perth Hospital (Practical Study EC 2004/030).

\section{Blood samples}

Venous blood was collected by clean venipuncture into vacuum tubes containing $0.109 \mathrm{M}$ buffered sodium citrate, $15 \mathrm{~mm}$ theophylline, $3.7 \mathrm{~mm}$ adenosine and $0.198 \mathrm{~mm}$ dipyridamole (CTAD tubes; Becton Dickinson, Franklin Lakes, NJ, USA) or $0.106 \mathrm{M}$ sodium citrate (Becton Dickinson). Samples were gently mixed by inversion.

\section{Controls}

Blood was collected from 20 healthy volunteers (10 males, range: $21-53$ years and 10 females, range: $21-$ 43 years), who had not taken aspirin or nonsteroidal anti-inflammatory drugs within the previous 10 days. These controls were used to establish normal ranges for activated platelets, PDM and platelet-monocyte complexes.

\section{Patients}

Patients were male or nonpregnant females aged at least 18 years of age presenting with unstable angina 
or non-ST-elevation myocardial infarction who underwent early percutaneous coronary intervention (PCI) within $48 \mathrm{~h}$ of presentation. They were excluded if they had active bleeding, a high risk of bleeding, had taken nonsteroidal anti-inflammatory drugs other than aspirin less than $48 \mathrm{~h}$ prior to presentation, and had been administered glycoprotein IIb/IIIa inhibitors, thienopyridines or dipyridamole within 7 days prior to presentation. Patients were given aspirin $300 \mathrm{mg}$ (if not already on aspirin) and clopidogrel (300 or $600 \mathrm{mg}$ ) at least $2 \mathrm{~h}$ prior to the procedure. Blood samples were collected postdose but prior to PCI. Heparin was administered for PCI, and in 17/20 patients abciximab, a glycoprotein IIb/IIIa inhibitor, was also administered with a bolus of $0.25 \mathrm{mg} / \mathrm{kg}$ followed by a continuous infusion of $0.25 \mu \mathrm{g} / \mathrm{kg} / \mathrm{min}$ post procedure. Further blood samples were collected the next day.

\section{Platelet-derived microparticles and activated platelets}

Fifty microlitres of whole blood was diluted with $950 \mu \mathrm{l}$ phosphate buffered saline $\mathrm{pH} 7.4 \quad(23.4 \mathrm{~g} / \mathrm{l}$ $\mathrm{NaH}_{2} \mathrm{PO}_{4} .2 \mathrm{H}_{2} \mathrm{O}, 21.3 \mathrm{~g} / \mathrm{l} \mathrm{Na} \mathrm{HPO}_{4}, 9.0 \mathrm{~g} / \mathrm{l} \mathrm{NaCl}$ ) containing $3.35 \mathrm{~g} / \mathrm{l}$ of dipotassium EDTA and $2 \mathrm{~g} / \mathrm{l}$ bovine albumin (PBS/EDTA/albumin buffer). Fifty microlitres of this diluted whole blood was incubated at room temperature for 15 min with $5 \mu \mathrm{l}$ murine monoclonal anti-CD61 (GPIIIa) labelled with fluorescein isothiocyanate (FITC) (DAKO, Glostrup, Denmark) and $5 \mu \mathrm{l}$ murine monoclonal anti-CD62 (P-selectin) labelled with R-phycoerythrin (RPE) (DAKO) and gently mixed. Following incubation, samples were fixed by adding $500 \mu \mathrm{l}$ of $1 \%$ paraformaldehyde (Sigma Chemical Co, St Louis, MO, USA) in PBS ( $\mathrm{pH}$ 7.4). Ten microlitres of fluorospheres (Coulter Corp., Miami, FL, USA) were added as an internal standard to quantitate platelet-derived microparticles. Samples were mixed by inversion and analysed using a Coulter EPICS XL-MCL flow cytometer (Coulter Corp). All antibodies were titrated to determine saturating concentrations.

Platelets were gated on CD61 positivity and activated platelets were detected by the presence of surface P-selectin (CD62). Results were expressed as a percentage of total platelets. Using flow beads of standard size (Flow Check; Polysciences Inc, Warring- ton, PA, USA) platelet-derived microparticles were characterized by size [less than $1 \mu \mathrm{m}$ (Heijnen et al., 1999)] and CD61 positivity (Figure 1). Isotype matched controls (DAKO) were used to correct for nonspecific binding. Data acquisition was stopped after 1000 fluorospheres were counted. To quantitate the concentration of PDM, the following calculation was used:

(Test Count-Isotype Control Count $) \times$ blood dilution $\times$ proportion of flow beads counted to total amount added. Results were reported as $\times 10^{8} / 1$.

\section{Detection of platelet-monocyte complexes}

Fifty microlitres of whole blood was diluted with $450 \mu \mathrm{l} \mathrm{PBS/EDTA/albumin} \mathrm{buffer.} \mathrm{Fifty} \mathrm{microlitres} \mathrm{of}$ this diluted whole blood was incubated at room temperature with $10 \mu \mathrm{l}$ murine monoclonal antibodies, anti-CD61-FITC, anti-CD45 R-phycoerythrin covalently linked to cyanine 5 (PeCy5) (Coulter, Immunotech, Marseille, France) and anti-CD14-RPE (DAKO) for $15 \mathrm{~min}$. After incubation, $500 \mu \mathrm{l}$ of $1 \%$ paraformaldehyde in distilled water was added as a fixative. Samples were gently mixed by inversion and analysed by flow cytometry.

Leucocytes were selected from red blood cells using the specific marker CD45 PeCy5. A scatter plot of forward $v s$. side scatter was used to identify the three distinct subpopulations of leucocytes. A gate was drawn around the monocyte population for further analysis. Cells positive for both anti-CD14 expression and CD61 expression were identified as platelet-monocyte complexes. (Figure 2). Cells were counted for $5 \mathrm{~min}$. Isotype-matched negative controls were performed to check for nonspecific binding. Results were expressed as a percentage of the total monocyte positive population.

\section{Stability studies}

Blood samples were processed for PDM, activated platelets and platelet-monocyte complexes immediately after collection and at $30 \mathrm{~min}, 1 \mathrm{~h}, 2 \mathrm{~h}$ and $6 \mathrm{~h}$ post venipuncture, in both CTAD and citrated tubes. In a separate experiment, blood samples fixed with $1 \%$ paraformaldehyde postincubation were read on the EPICS XL-MCL flow cytometer at 1 -h intervals for $5 \mathrm{~h}$. 


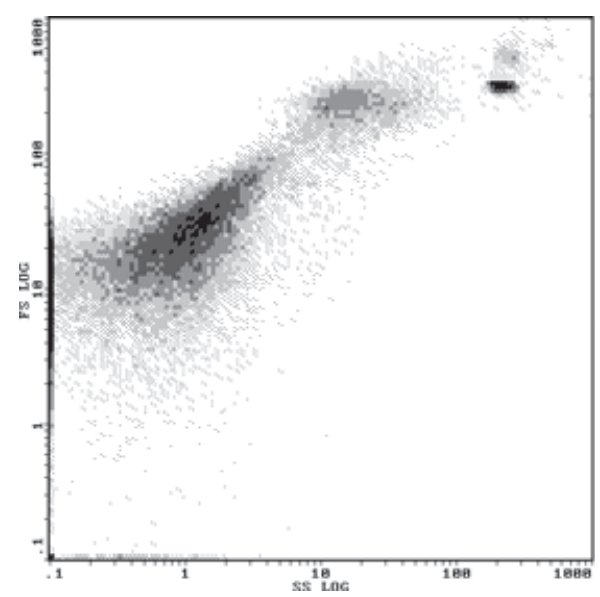

Histogram 1

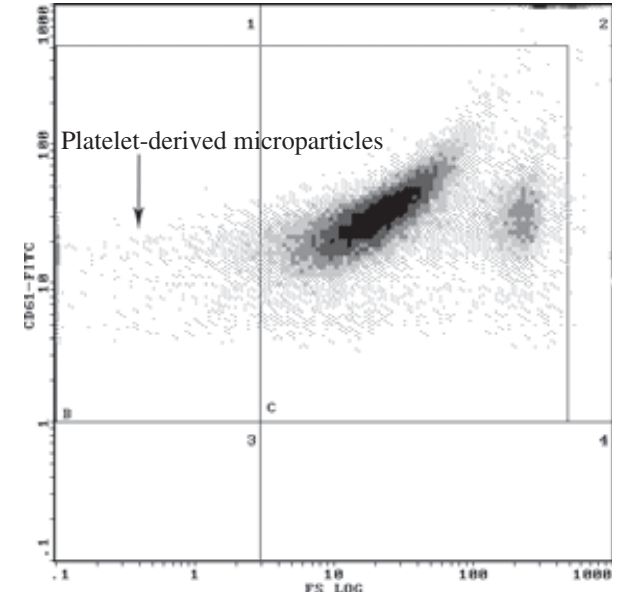

Histogram 2

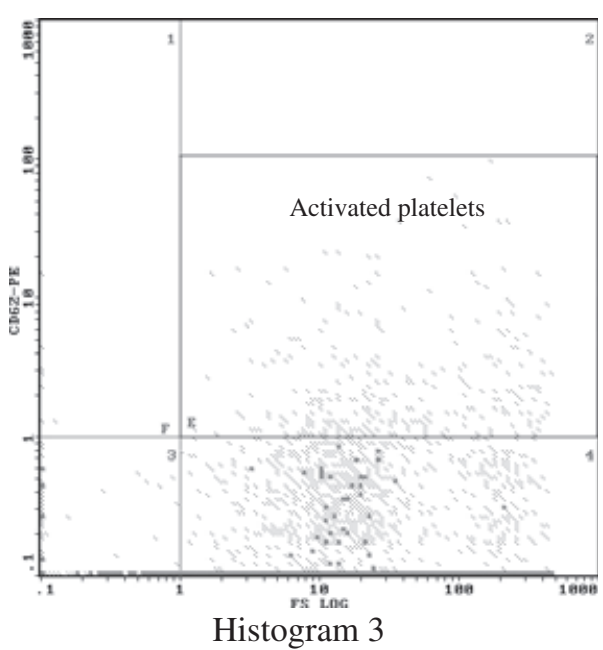

Figure 1. Flow cytometric analysis of platelet-derived microparticles (PDM) and activated platelets. Platelets are discriminated from leucocytes and erythrocytes based on typical forward scatter (FS) and side scatter (SS) properties in histogram 1. PDM are characterized by their size and positivity for CD61-FITC and FS in histogram 2. Platelets with surface P-selectin expression (CD62) can be seen in histogram 3.

\section{Detection of activation by in vitro stimulation}

Citrated whole blood was stimulated in vitro with a final concentration of $1 \mu \mathrm{g} / \mathrm{ml}$ of collagen (Helena Laboratories, Mt Waverley, Australia) at $37{ }^{\circ} \mathrm{C}$ for $15 \mathrm{~min}$. Samples were processed pre- and post activation for PDM, activated platelets and platelet-monocyte complexes.

\section{Plasma markers of platelet activation}

Citrated blood samples were spun at $1300 \mathrm{~g}$ for $10 \mathrm{~min}$ and plasma aliquots were snap frozen in liquid nitrogen and stored at $-80{ }^{\circ} \mathrm{C}$ until assayed. Commercial sandwich ELISA kits (Bender MedSystems, San Bruno, CA, USA) were used to quantify plasma platelet activation markers, soluble CD40 ligand and soluble P-selectin.

\section{Plasma procoagulant phospholipid}

Citrated blood samples were spun at $1300 \mathrm{~g}$ for $10 \mathrm{~min}$ and plasma aliquots were snap frozen in liquid nitrogen and stored at $-80{ }^{\circ} \mathrm{C}$ until assayed. Centrifuged plasmas had residual platelet counts of $<3 \times 10^{9} / 1$. A commercial kit (XACT, Haematex Research, Hornsby, Austra- 


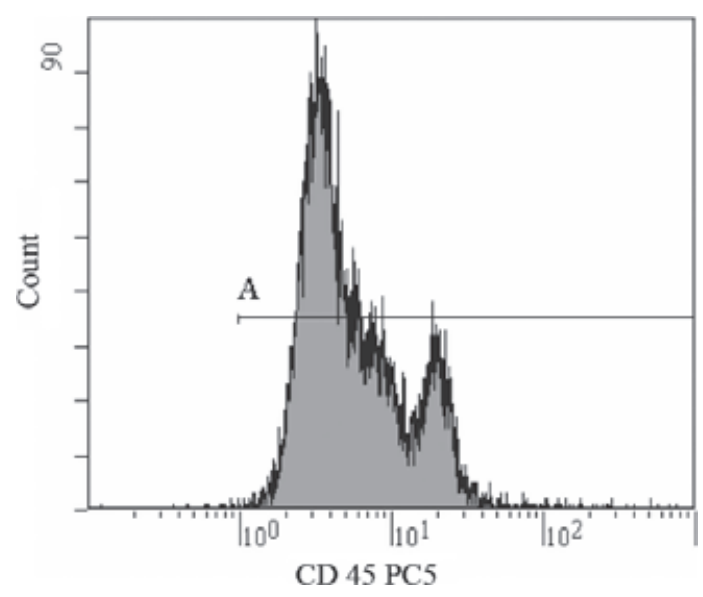

Histogram 1

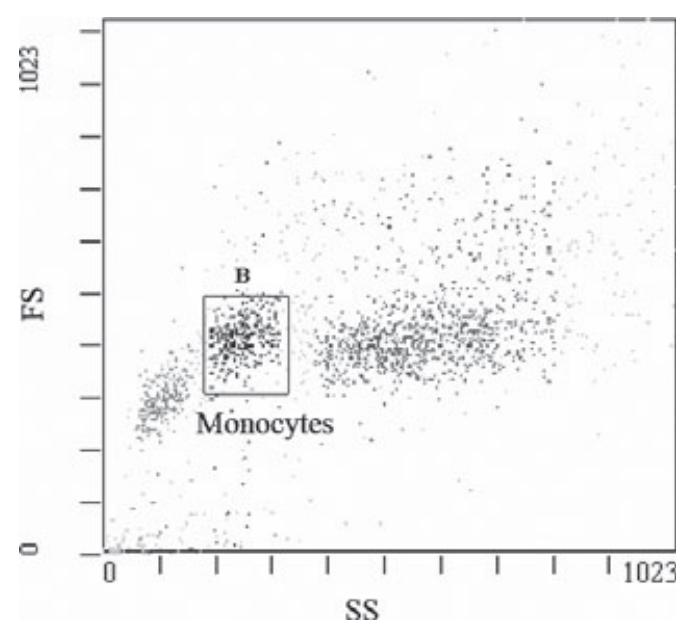

Histogram 2

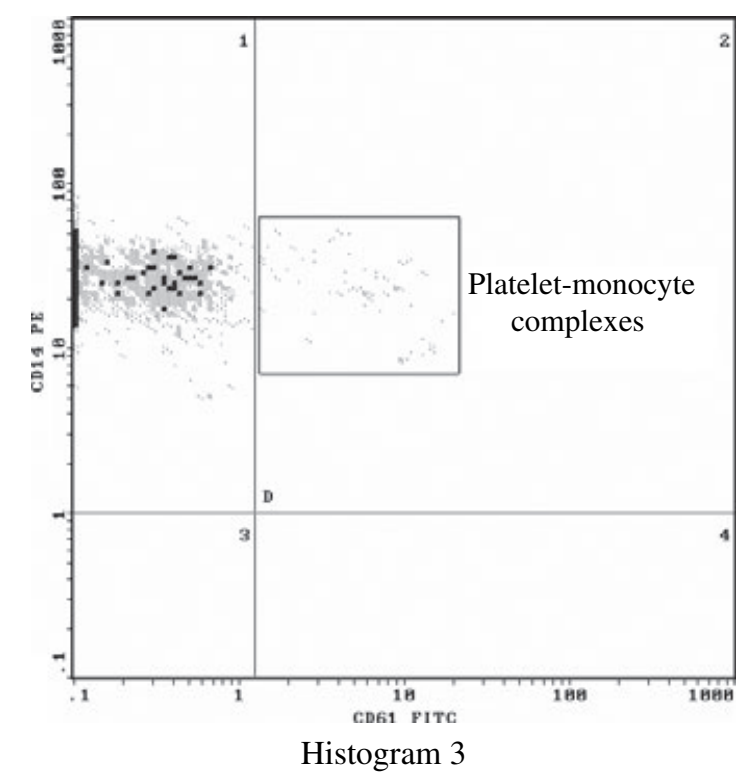

Figure 2. Flow cytometric analysis of platelet-monocyte aggregates in whole blood. The instrument is triggered by the fluorescence signal and CD45-PeCy5 positive events (leucocytes) and are gated by A in histogram 1 . These are then discriminated by their forward scatter (FS) and side scatter (SS) characteristics in histogram 2, monocytes are discriminated by rectangle B. Events presenting in B are considered monocytes and are subjected to two-colour analysis (CD61-FITC and CD14-RPE) in histogram 3 for the discrimination of platelet-monocyte complexes.

lia) was used to quantify procoagulant phospholipid. This is a clotting technique with phospholipid free porcine plasma and activated factor $\mathrm{X}$. The phospholipid in the patient plasma shortens the clotting time and is the rate limiting factor. The assay was performed on the STAR automated coagulation analyser (Diagnostica Stago, Asnières, France).

\section{Statistical methods}

Data are presented as median and range. Data normally distributed were compared by a Student's $t$-test. Mann-Whitney $U$-test (unrelated groups) and the Wilcoxon signed ranks test (two related samples) were used to assess non-normally distributed data.

\section{(c) 2008 The Authors}


A $P$ value of $<0.05$ was considered statistically significant.

\section{RESULTS}

\section{Stability studies}

Platelet-derived microparticles levels were stable for $2 \mathrm{~h}$ in both citrate and CTAD tubes but increased by $1.8 \%$ in citrate tubes and $1.49 \%$ in CTAD tubes after $6 \mathrm{~h}$ (mean of five experiments). Activated platelets increased from $0.45 \%$ immediately after collection to $1.06 \%$ after $2 \mathrm{~h}$ and $3.59 \%$ after $6 \mathrm{~h}$ in citrate tubes and at the same time-point levels were $0.29,0.31$ and $3.84 \%$, respectively, in CTAD tubes (mean of five experiments). Platelet-monocyte complexes rose from a baseline 3.05 to $5.06 \%$ at $2 \mathrm{~h}$ and $11.5 \%$ at $6 \mathrm{~h}$ in citrate anticoagulant and were 3.11, 4.15 and $10.4 \%$, respectively, in CTAD (mean of five experiments). For the remainder of the study, sodium citrate tubes were processed within $30 \mathrm{~min}$ or CTAD tubes within $2 \mathrm{~h}$ post venepuncture.

The effect of fixing whole blood with $1 \%$ paraformaldehyde was examined over a 6-h period. All samples were stable for $2 \mathrm{~h}$ post fixation in the fridge (data not shown).

\section{Detection of activation poststimulation}

In an unstimulated sample PDM, activated platelets and platelet-monocyte complexes were $184 \times 10^{8} / \mathrm{l}$, 0.87 and $2.71 \%$, respectively. Upon stimulation with a final concentration of $1 \mu \mathrm{g} / \mathrm{ml}$ of collagen at $37{ }^{\circ} \mathrm{C}$ for $15 \mathrm{~min}$, all markers of platelet activation increased; PDM $434 \times 10^{8} / \mathrm{l}$, activated platelets $40.1 \%$ and platelet-monocyte complexes $17.1 \%$. With final collagen concentrations of 5 and $10 \mu \mathrm{g} / \mathrm{ml}$, the results were PDM 492 and $726 \times 10^{8} / \mathrm{l}$, activated platelets 59.6 and $89.4 \%$ and platelet-monocyte complexes 71.3 and $86.1 \%$, respectively.

\section{Reproduceability}

The intra-assay coefficient of variation for the same sample 5 consecutive times was $5.3 \%$ for PDM, $10.9 \%$ for activated platelets and $13.3 \%$ for plateletmonocyte complexes.

\section{Normal values}

Twenty healthy volunteers were assessed for all markers of platelet activation. The results for PDM ranged from 38 to $216 \times 10^{8} / \mathrm{l}$, activated platelets $0.1-1.03 \%$ and platelet-monocyte complexes $0.64-7.48 \%$. The results for this group are summarized in Table 1. The results of the plasma markers soluble P-selectin, soluble CD40 ligand and procoagulant phospholipid XACT test are shown in Table 2.

\section{Patients}

Activated platelets and platelet-monocyte complexes were higher in the pre-PCI samples compared with the normal values. After the procedure, circulating

Table 1. Cellular markers of platelet activation in normal volunteers and patients pre- and postpercutaneous coronary intervention (PCI)

\begin{tabular}{|c|c|c|c|c|c|c|}
\hline & \multirow[b]{2}{*}{ Normal } & \multirow[b]{2}{*}{ Pre-PCI } & \multirow[b]{2}{*}{ Post-PCI } & \multicolumn{3}{|l|}{$P$-value } \\
\hline & & & & $\begin{array}{l}\text { Normal } v s . \\
\text { pre-PCI }\end{array}$ & $\begin{array}{l}\text { Normal vs. } \\
\text { post-PCI }\end{array}$ & $\begin{array}{l}\text { Pre-PCI vs. } \\
\text { post-PCI }\end{array}$ \\
\hline $\begin{array}{l}\text { Platelet derived } \\
\text { microparticles }\left(\times 10^{8} / 1\right)\end{array}$ & $106(42-216)$ & $83(38-212)$ & $90(28-265)$ & NS & NS & NS \\
\hline Activated platelets (\%) & $0.39(0.11-1.03)$ & $0.95(0.05-3.48)$ & $0.57(0.25-1.77)$ & 0.005 & NS & 0.0027 \\
\hline $\begin{array}{l}\text { Platelet monocyte } \\
\text { complexes }(\%)\end{array}$ & $2.98(0.64-7.48)$ & $4.33(1.73-10.60)$ & $3.91(2.07-13.4)$ & 0.0275 & 0.0349 & NS \\
\hline
\end{tabular}

There was a significant increase in activated platelets and platelet monocyte complexes between pre-PCI patients and normal samples. The data presented are median (range). NS, not significant. 
Table 2. Plasma markers of platelet activation in normal volunteers and patients pre- and postpercutaneous coronary intervention (PCI)

\begin{tabular}{|c|c|c|c|c|c|c|}
\hline & \multirow[b]{2}{*}{ Normal } & \multirow[b]{2}{*}{ Pre-PCI } & \multirow[b]{2}{*}{ Post-PCI } & \multicolumn{3}{|l|}{$P$-value } \\
\hline & & & & $\begin{array}{l}\text { Normal } v s . \\
\text { pre-PCI }\end{array}$ & $\begin{array}{l}\text { Normal } v s . \\
\text { post-PCI }\end{array}$ & $\begin{array}{l}\text { Pre-PCI vs. } \\
\text { post-PCI }\end{array}$ \\
\hline $\begin{array}{l}\text { XACT (plasma } \\
\text { phospholipid } \mu \mathrm{g} / \mathrm{ml} \text { ) }\end{array}$ & $1.87(0.7-3.55)$ & $1.3(0.58-2.43)$ & $0.68(0.14-2.56)$ & NS & $<0.0001$ & 0.0032 \\
\hline sP-selectin (ng/ml) & $30.7(22.3-69.7)$ & $34.14(27.95-95.72)$ & $28.77(19.71-51.14)$ & NS & NS & 0.0066 \\
\hline SCD40 ligand $(\mathrm{ng} / \mathrm{ml})$ & $0.46(0.17-3.82)$ & $0.30(0.21-1.69)$ & $0.24(0.03-1.61)$ & NS & 0.0006 & $<0.0001$ \\
\hline
\end{tabular}

There was a significant fall in all markers post-PCI. Data presented are median (range).

$\mathrm{XACT}$, activated Factor $\mathrm{X}$ assay for procoagulant phospholipid; sP-selectin, soluble P-selectin; SCD40L, soluble CD40 Ligand; NS, not significant.

activated platelets were no longer significantly different to normal. Platelet-monocyte complexes were still increased although less significantly so. Pre-PCI PDM values were not significantly different to normal and were unchanged post-PCI. (Table 1).

There was no significant difference in soluble platelet activation markers between normal and patient pre-PCI. Post-PCI levels of plasma phospholipid and sCD40 ligand were significantly lower than normal values and all three plasma markers were significantly lower post-PCI vs. pre-PCI. (Table 2).

\section{DISCUSSION}

Circulating activated platelets have been associated with many common disorders including stable and unstable angina (Vidal et al., 2001), acute myocardial infarction (Furman et al., 2001a), diabetes mellitus (Nomura et al., 1995), angioplasty (Michelson et al., 2001), cardiopulmonary bypass (Nannizzi-Alaimo et al., 2002), acute cerebral ischaemia (Zeller, Tschoepe \& Kessler, 1999), myeloproliferative disorders (Wehmeier et al., 1991) and peripheral arterial disease (Cassar et al., 2003).

Flow cytometry is a versatile tool that can be utilized to quantitatively assess the physical and antigenic properties of platelets (Linden et al., 2004). A major challenge in analysing the activation status of circulating platelets is to avoid artefactual in vitro activation. The technique reported here minimizes artefactual trauma by testing in whole blood, avoiding red blood cell lysis, centrifugation and washing steps. The samples are fixed with $1 \%$ paraformaldehyde postan-

(C) 2008 The Authors

Journal compilation (C) 2008 Blackwell Publishing Ltd, Int. Jnl. Lab. Hem. tibody labelling to increase the sample stability. The method is rapid, easy to perform and simultaneously measures a panel of platelet activation markers.

Sodium citrate is the most commonly used anticoagulant for platelet activation studies (Schmitz et al., 1998). This work shows that citrate samples need to be processed within $30 \mathrm{~min}$ of venepuncture. CTAD anticoagulant contains platelet inhibitors to minimize ex vivo activation (Mody et al., 1999; Kim et al., 2002). We found samples collected into these tubes were stable for $2 \mathrm{~h}$ postvenesection.

The intrabatch coefficient of variation (CV) was below $15 \%$ for all markers. The technique was sensitive to small incremental increases over time and easily detected the activation changes induced by collagen stimulation.

Platelet-monocyte aggregates have been reported to be a more sensitive marker of in vivo platelet activation than surface P-selectin expression (Michelson et al., 2001). There are two approaches to identify platelet-monocyte complexes. First is to use cell separation and red blood cell lysis to remove erythrocytes and allow clear differentiation of white cell populations. (Li, Goodall \& Hjemdahl, 1997; Michelson et al., 2001; Barnard et al., 2003). An alternative technique is to avoid lysis thus minimizing platelet activation (Hagberg \& Lyberg, 2000). In the presence of intact red cells, leucocytes can be discriminated from erythrocytes by binding of the leucocyte specific antiCD45. A combination of a platelet-specific antibody anti-CD61 and a monocyte-specific antibody, antiCD14 allows the detection of platelet-monocyte complexes (Barnard et al., 2003). 
Normal reference ranges were determined from 20 healthy volunteers. The reference range for plateletmonocyte complexes was lower than reported by some other authors. Ray et al. (2005) reported a reference range of $3.2-12.4 \%$ compared with the reference range in this study of $0.64-7.48 \%$. The lower reference range of this study may be due to the avoidance of red cell lysis. The reference range for activated platelets (P-selectin expression) in this study correlates with several other studies (Furman et al., 1998; McCabe et al., 2004; Ray et al., 2005). The absolute values for PDM obtained in this study are higher than those found in plasma (Kim et al., 2002) most likely because of the lack of a centrifugation step.

Platelets have a key role in the pathogenesis of arterial thrombosis and acute coronary syndromes (Vidal et al., 2001). This study demonstrates a significant degree of platelet activation in patients prior to PCI when compared with the normal population demonstrated by increased platelet expression of surface P-selectin and platelet-monocyte complexes and correlates well with several other studies (Furman et al., 2001a; Vidal et al., 2001; Craft et al., 2004; Morel et al., 2004; Ray et al., 2005). Soluble markers of platelet activation, CD40 ligand and P-selectin, were not significantly different from the normal population in pre-PCI patients, suggesting flow cytometric analysis may offer a more sensitive measure of in vivo platelet activation.

Postpercutaneous coronary intervention is an invasive procedure that results in endothelial trauma and can contribute to restenosis (Craft et al., 2004; Ray et al., 2005). Thus, there is an increased need for adjunctive therapy. Following PCI, there were significant decreases in activated platelets, soluble P-selectin, procoagulant phospholipid and soluble CD40L. This can be attributed to antiplatelet and anticoagulant therapy administered prior to, and during, the procedure. Several studies have reported that the use of GPIIbIIIa inhibitors, oral anticoagulants and aspirin to decrease platelet activation after PCI (Mascelli et al., 1998; Furman et al., 2001b; Berg et al., 2002a,b; Ray et al., 2005).

Platelet-monocyte complexes, however, remained elevated after PCI and antiplatelet therapy. This is likely to be due to the dynamic interaction of heparin anticoagulation and antiplatelet therapy. Michelson et al. (2001) reported platelet-monocyte complexes increased immediately post-PCI but returned to baseline $24 \mathrm{~h}$ post-PCI, whereas Furman et al. (2001b) noted that unfractionated heparin increased plateletmonocyte complexes. In contrast, two studies have reported that the use of GPIIbIIIa inhibitors lowers platelet-monocyte complexes (Furman et al., 200lb; Ray et al., 2005).

Several groups have shown an increase in SCD40L in patients requiring PCI (Aukrust et al., 1999; Srinivasa Prasad et al., 2003) as well as post-PCI (Quinn et al., 2004). However, this study found significantly decreased levels in both groups when compared with normal samples. Quinn et al. (2004) reported clopidogrel treatment prior to PCI had no effect on elevated serum CD40L levels post-PCI. The difference in these results may be due to sample type. Thom et al. (2004) reported that SCD40L is significantly higher in serum when compared with citrated plasma and the degree of elevation in serum is proportional to the time elapsed after venepuncture until the sample has fully clotted. Citrated plasma is more likely to represent in vivo physiological levels of soluble CD40L.

During platelet activation, anionic phospholipids are exposed to the platelet surface and PDM are formed creating a procoagulant surface. This is the matrix for the reaction of factor Xa, thrombin and fibrin clot formation (Holme, Brosstad \& Solum, 1995). The XACT test is a new method that quantitates procoagulant phospholipid in plasma (Exner et al., 2003). Test plasma is mixed with phospholipid free porcine plasma and activated factor $\mathrm{X}$. Anionic phospholipid in the patient plasma is rate limiting and will shorten clotting in proportion to its concentration. The method is easily automated and the clotting time in seconds is converted to a concentration of phospholipid by extrapolation from a standard curve. This technique demonstrated a significant decrease in procoagulant phospholipid post-PCI which mirrored the decrease in activation observed in other markers of platelet activation such as sP-selectin, sCD40 ligand and activated platelets. This may be a useful rapid method to measure platelet activation in plasma. Interestingly, there was not a corresponding decrease in PDM suggesting the relationship between PDM and plasma phospholipid in whole blood may be more complex than simply numerical.

This paper reports a whole blood flow cytometric technique for the detection of PDM, activated platelets

(c) 2008 The Authors

Journal compilation (c) 2008 Blackwell Publishing Ltd, Int. Jnl. Lab. Hem. 
and platelet-monocyte complexes, which offers the advantage of minimal sample manipulation avoiding artefactual platelet activation. Using the technique, we have developed normal ranges for PDM, activated platelets and platelet monocyte complexes. We examined a group of patients pre- and post-PCI and found evidence of activation prior to procedure and a marked decrease in platelet activation post-PCI, suggesting effectiveness of clinical intervention. A limitation of this study is the small number of patients analysed. Larger prospective studies are required to confirm the decrease in platelet activation markers post-PCI with adjunctive therapy.

The morbidity and mortality caused by thrombosis far exceeds that as a result of bleeding diatheses. A platelet activation profile that includes the enumeration of PDM, activated platelets and platelet-monocyte complexes may be useful to predict the risk of thrombosis, monitor antiplatelet therapy and assess the effectiveness of clinical intervention.

\section{REFERENCES}

Andre P. (2004) P-selectin in haemostasis. British Journal of Haematology 125 , 298-306.

Aukrust P., Muller F., Ueland T., Berget T., Aaser E., Brunsvig A., Solum N., Forfang K., Froland S. \& Gullestad L. (1999) Enhanced levels of soluble and membrane-bound CD40L in patients with unstable angina: possible refelction of $\mathrm{T}$ lymphocyte and platelet involvement in the pathogensis of acute coronary syndromes. Circulation 100, 614620.

Barnard M., Krueger L.A., Frelinger A., III, Furman M. \& Michelson A. (2003) Whole blood analysis of leukocyteplatelet aggregates. In Current Protocols in Cytometry, pp. 6.15.11-6.15.18. John Wiley and Sons.

Berg J., Gerristen W., Haas F., Kelder H., Verheugt F. \& Plokker H. (2002a) Highdose aspirin in addition to daily lowdose aspirin dcreases activation in patients before and after percutaneous coronary intervention. Thrombosis Research 105, 385-390.

Berg J., Gerristen W., Haas F., Kelder J., Verheugt F. \& Plokker H. (2002b) Pretreatment with oral anticoagulanys decreased platelet activaiton before and after percutaneous coronary intervention. Thrombosis and Haemostasis 88, 924-930

Cassar K., Bachoo P., Ford I., Greaves M. \& Brittenden J. (2003) Platelet activation is increased in peripheral arterial disease. Journal of Vascular Surgery 38, 99-103.

Craft J., Masci P., Roberts M., Brighton T., Garrahy P., Cox S. \& Marsh N. (2004) Increased platelet-derived microparticles in the coronary circulation of percutaneous transluminal coronary angioplasty patients. Blood Coagulation and Fibrinolysis 15, 475-482.

Ejim O., Powling M., Dandone P., Kernoff P. \& Goodall A. (1990) A flow cytometric analysis of fibronectin binding to platelets from patients with peripheral vascular disease. Thrombosis Research 58, 519-524.

Exner T., Joseph J., Low J., Connor D. \& Ma D. (2003) A new activated factor Xbased clotting method with improved specificity for procoagulant phospholipid. Blood Coagulation and Fibrinolysis $14,773-779$.

Furman M.I., Benoit S.E., Barnard M.R., Valeri C.R., Borbone M.L., Becker R.C., Hechtman H.B. \& Michelson A.D. (1998) Increased platelet reactivity and circulating monocyte-platelet aggregates in patients with stable coronary artery disease. Journal of American College of Cardiology 31, 352-358.

Furman M.I., Barnard M.R., Krueger L.A., Fox M.L., Shilale E.A., Lessard D.M., Marchese P., Frelinger A.L., III, Goldberg R.J. \& Michelson A.D. (200la) Circulating monocyte-platelet aggregates are an early marker of acute myocardial infarction. Journal of the American College of Cardiology 38, 1002-1006.

Furman M.I., Kereiakes D., Krueger L.A., Mueller M., Pieper K., Broderick T., Schneider J., Howard W., Fox M.L., Barnard M.R., Frelinger A.L., III $\delta$ Michelson A.D. (2001b) Leukocyteplatelet aggregation, platelet surface Pselectin, and platelet surface glycoprotein IIIa after percutaneous coronary intervention: Effects of dalteparin or unfractionated heparin in combination with abciximab. American Heart Journal 142, 790-798.

Hagberg I.A. \& Lyberg T. (2000) Evaulation of circulating platelet-leukocyte conjugates: a sensitive flow cytometric assay well sutied for clinical studies. Platelets 11, 151-160.

Heijnen H.F., Schiel A.E., Fijnheer R., Geuze H.J. \& Sixma J.J. (1999) Activated platelets release two types of membrane vesicles: microvesicles by surface shedding and exosomes derived from exocytosis of multivesicular bodies and alphagranules. Blood 94, 3791-3799.

Hickerson D.H.M. \& Bode A.P. (2002) Flow cytometry of platelets for clinical analysis. Hematology/Oncology Clinics of North America 16, 421-454.

Holme P.A., Brosstad F. \& Solum N.O. (1995) Platelet-derived microvesicles and activated platelets express factor Xa activity. Blood Coagulation and Fibrinolysis $6,302-310$.

Kim H.K., Song K.S., Lee E.S., Lee Y.J., Park Y.S., Lee K.R. \& Lee S.N. (2002) Optimized flow cyometric assay for the measurement of platelet microparticles in plasma: pre-analytic and analytic considerations. Blood Coagulation and Fibrinolysis 13, 393-397.

Li N., Goodall A.H. \& Hjemdahl P. (1997) A sensitive flow cytometric assay for circulating platelet-leucocyte aggregates. British Journal of Haematology 99, 808-816.

Linden M.D., Frelinger A.L., III, Barnard M.R., Przyklenk K., Furman M.I. \& Michelson A.D. (2004) Application of flow cytometry to platelet disorders. Seminars in Thrombosis and Hemostasis 30, 501-511.

Mascelli M.A., Lance E.T., Damaraju L., Wagner C.L., Weisman H.F. \& Jordan 
R.E. (1998) Pharmacodynamic profile of short-term Abciximab treatment demonstrates prolonged platelet inhibition with gradual recovery from GP IIb/IIIa receptor blockade. Circulation 97, 1680-1688.

McCabe D.J.H., Harrison P., Mackie I.J., Sidhu P.S., Purdy G., Lawrie A.S., Watt H., Brown M.M. \& Machin S.J. (2004) Platelet degranulation and monocyteplatelet complex formation are increased in the acute and convalescent phases after ischaemic stroke or transient ischaemic attack. British Journal of Haematology 125, 777-787.

Michelson A., Barnard M., Krueger L.A., Valeri C. \& Furman M. (2001) Circulating monocyte-platelet aggregates are a more sensitive marker of in vivo platelet activation than platelet surface P-selectin: studies in baboons, human coronary intervention and human acute myocardial infarction. Circulation 104, 1533-1537.

Mody M., Lazarus A.H., Semple J.W. \& Freedman J. (1999) Preanalytical requirements for flow cytometric evaluation of platelet activation: choice of anticoagulant. Transfusion Medicine 9, 147-154.

Morel O., Hugel B., Jesel L., Mallat Z., Lanza F., Douchet M., Zupan M., Chauivn M., Cazenave J., Tedgui A., Freyssinet J. \& Toti F. (2004) Circulating procoagulant microparticles and soluble GPV in myocaridal infarction treated by primary percutaneous transluminal cor- onary angioplasty. A possible role for GPIIb-IIIa antagonists. Journal of Thrombosis and Haemostasis 2, 1118 11126.

Nannizzi-Alaimo L., Rubenstein M.H., Alves V.L., Leong G.Y., Phillips D.R. \& Gold H.K. (2002) Cardiopulmonary bypass induces release of soluble CD40 Ligand. Circulation 105, 2849-2854.

Nomura S., Masahiko S., Katsura K., Xie G.L., Miyazaki Y., Miyake T., Kido H., Kagawa H. \& Fukuhara S. (1995) Platelet-derived microparticles may influence the development of atherosclerosis in diabetes mellitus. Atherosclerosis 116, 235-240.

Quinn M., Bhatt D., Zidar F., Vivekananthan D., Chew D., Ellis S., Plow E. \& Topol E. (2004) Effect of clopidogrel pretreatment on inflammatory marker expression in patients undergoing percutaneous coronary intervention. American Journal of Cardiology 93, 679-684.

Ray M., Walters J., Cameron B., Wood P. \& Aroney C. (2005) Platelet-monocyte aggregates predict troponin rise after percutaneous coronary intervention and are inhibited by Abciximab. Internation Journal of Cardiology 101, 249-255.

Schmitz G., Rothe G., Ruf A., Barlage S., Tschoepe D., Clementson K.J., Goodall A.H., Michelson A.D., Nurden A.T. \& Shankey T.V. (1998) European working group on clinical cell analysis: consensus protocol for the flow cytometric characterisation of platelet function.
Thrombosis and Haemostasis 79, 885896.

Shattil S.J., Cunningham M. \& Hoxie J.A. (1987) Detection of activated platelets in whole blood using activation-dependent monoclonal antibodies and flow cytometry. Blood 70, 307-315.

Prasad K., Andre P., Yan Y. \& Phillips D.R. (2003) The platelet CD40L/GP IIbIIIa axis in atherothrombotic disease. Current Opinion in Hematology 10, 356-361.

Thom J., Gilmore G., Yi Q., Hankey G.J. \& Eikelboom J. (2004) Measurement of soluble P-selectin and soluble CD40 ligand in serum and plasma. Journal of Thrombosis and Haemostasis 2, 1-2.

Vidal C., Spaulding C., Picard F., Schaison F., Melle J., Weber S. \& Fontenay-Roupie M. (2001) Flow cytometry detection of platelet procoagulant activity and microparticles in patients with unstable angina treated by percutaneous coronary angiplasty and stent implantation. Thrombosis and Haemostasis 86, 784790.

Wehmeier A., Tschope D., Esser J., Menzel C., Nieuwenhuis H.K. \& Schneider W. (1991) Circulating activated platelets in myeloproliferative disorders. Thrombosis Research 61, 271-278.

Zeller J.A., Tschoepe D. \& Kessler C. (1999) Circulating platelets show increased activation in patients with acute cerebral ischemia. Thrombosis and Haemostasis 81, 373-377. 